\title{
Genome-scale metabolic reconstruction and in silico analysis of methylotrophic yeast Pichia pastoris for strain improvement
}

\author{
Bevan KS Chung 1,2, Suresh Selvarasu², Andrea Camattari2, Jimyoung Ryu33, Hyeokweon Lee³, Jungoh Ahn³, \\ Hongweon Lee ${ }^{3}$ and Dong-Yup Lee*1,2,4
}

\begin{abstract}
Background: Pichia pastoris has been recognized as an effective host for recombinant protein production. A number of studies have been reported for improving this expression system. However, its physiology and cellular metabolism still remained largely uncharacterized. Thus, it is highly desirable to establish a systems biotechnological framework, in which a comprehensive in silico model of P. pastoris can be employed together with high throughput experimental data analysis, for better understanding of the methylotrophic yeast's metabolism.

Results: A fully compartmentalized metabolic model of P. pastoris (iPP668), composed of 1,361 reactions and 1,177 metabolites, was reconstructed based on its genome annotation and biochemical information. The constraints-based flux analysis was then used to predict achievable growth rate which is consistent with the cellular phenotype of $P$. pastoris observed during chemostat experiments. Subsequent in silico analysis further explored the effect of various carbon sources on cell growth, revealing sorbitol as a promising candidate for culturing recombinant $P$. pastoris strains producing heterologous proteins. Interestingly, methanol consumption yields a high regeneration rate of reducing equivalents which is substantial for the synthesis of valuable pharmaceutical precursors. Hence, as a case study, we examined the applicability of P. pastoris system to whole-cell biotransformation and also identified relevant metabolic engineering targets that have been experimentally verified.

Conclusion: The genome-scale metabolic model characterizes the cellular physiology of P. pastoris, thus allowing us to gain valuable insights into the metabolism of methylotrophic yeast and devise possible strategies for strain improvement through in silico simulations. This computational approach, combined with synthetic biology techniques, potentially forms a basis for rational analysis and design of P. pastoris metabolic network to enhance humanized glycoprotein production.
\end{abstract}

\section{Background}

In the biopharmaceutical industry, over $70 \%$ of the therapeutic proteins under preclinical and clinical development are glycosylated and there has been an increasing need for highly efficient glycoprotein expression systems. Mammalian systems such as Chinese hamster ovary (CHO) cells have been most widely used since they have been extensively characterized and are capable of humanlike glycosylation. However, they typically exhibit low survivability and low recombinant protein productivity

*Correspondence: cheld@nus.edu.sg

1 NUS Graduate School for Integrative Sciences and Engineering, National University of Singapore, 28 Medical Drive, \#05-01, 117456, Singapore Full list of author information is available at the end of the article unless sophisticated experimental techniques were employed [1]. On the other hand, although yeast systems typically produce hyper-mannosylated proteins with poor bioactivity in humans, recent advances in yeast glycoengineering, especially for Pichia pastoris, enabled the synthesis of humanized glycoproteins with substantially improved bioactivity $[2,3]$. Furthermore, since the scaleup of production in yeast is a well-established technology, we can potentially achieve cost-effective and highthroughput production of therapeutic glycoproteins $[2,3]$. Thus, the methylotrophic yeast $P$. pastoris is expected to be one of the promising hosts for industrial production of recombinant protein in the near future. Indeed, compa- 
nies are already beginning to use $P$. pastoris for the production of several therapeutic proteins $[4,5]$.

A number of studies have been carried out for understanding and enhancing physiological properties of $P$. pastoris. These studies have elucidated various favorable characteristics of the organism, which makes it more attractive for large-scale production of recombinant humanized glycoprotein [6-10]. In particular, the lower tendency of hyper-mannosylation of proteins and negative Crabtree phenotype can present $P$. pastoris as a more superior expression host than the well-characterized Saccharomyces cerevisiae $[7,11]$. Hence, it is highly desirable to make significant efforts for improving the P. pastoris strain in order to achieve economic feasibility and efficiency [12-14]. In this regard, the emerging paradigm of systems biotechnology can play an important role in identifying key targets for strain improvement [15].

Systems biotechnology integrates high-throughput omics data and in silico modeling and analysis to understand and design cellular system to achieve desirable properties [16]. This approach has been successfully applied to one of the best-characterized expression system, S. cerevisiae, which was metabolically engineered for improved production of chemicals and biopharmaceuticals $[17,18]$. For example, targets for genetic manipulation have been identified to enhance the production of bioethanol $[19,20]$ and human superoxide dismutase [21]. Therefore, we can yield similar benefits for P. pastoris by resorting to the systems biotechnological approach, which requires a comprehensive in silico metabolic model [22].

To date, more than 50 genome-scale metabolic models have been reconstructed for over 30 species from three main domains of life, i.e. Archaea, Bacteria and Eukarya $[23,24]$. Various applications of genome-scale metabolic models have been reported for characterizing cellular metabolism and guiding metabolic engineering. Examples include characterization of various organisms ranging from unicellular bacteria such as Escherichia coli [25] and Zymomonas mobilis (Widiastuti H, Kim JY, Selvarasu S, Karimi IA, Kim H, Seo JS, Lee DY: Genome-scale modeling and in silico analysis of ethanologenic bacteria Zymomonas mobilis, submitted) to complex mammalian systems such as Mus musculus [26] and even Homo sapiens [27]; phenotypic prediction of metabolic-gene deletion strains of $E$. coli [28]; and identification of metabolic engineering targets to enhance biochemical production capability of $E$. coli $[29,30]$. Towards this end, we reconstructed a genome-scale model of $P$. pastoris metabolism which allows us to elucidate interesting features of the methylotrophic yeast and identify engineering targets for achieving enhanced physiological properties of the strain.

\section{Results}

Characteristics of reconstructed metabolic network

Following the procedure illustrated in Figure 1, a genome-scale network of $P$. pastoris metabolism was reconstructed (see Methods). During the reconstruction process, significant efforts were required in the gap-filling process. Essential metabolic reactions are added sequentially to the draft model, through repeated cell growth simulations using constraints-based flux analysis, to ensure in silico viability. For example, a metabolic gap exists in the initial draft of the methonine biosynthetic pathway as the gene coding for the enzyme catalyzing transamination of 2-keto-4-methylbutyrate to form methionine [31] was not found and had to be added manually. Similarly, in order to allow mitochondrial localization of acyl-carrier protein for fatty acid synthesis, a mitochondrial transport reaction was introduced to the model, thus improving metabolic network connectivity.

The reconstructed metabolic model of $P$. pastoris, iPP668, accounts for 668 metabolic genes, 1,361 reactions and 1,177 metabolites segregated into 8 compartments: cytosol, endoplasmic reticulum, extracellular matrix, Golgi apparatus, mitochondria, nucleus, peroxisome and vacuole as summarized in Table 1 (see Additional file 1 for model details). The metabolic reactions include 904 intra-compartment reactions, 308 inter-compartment reactions, and 149 extracellular exchange reactions. These metabolic reactions are also classified into 62 subsystems (Table 2) which include pathways in central carbon metabolism, amino acid, carbohydrate and lipid biosynthetic pathways, and more notably, the methanol utilization pathway previously characterized by [32]. In the fully compartmentalized model, metabolites localized in different cellular compartments (e.g. cytosolic pyruvate, $\operatorname{pyr}[\mathrm{c}]$ and mitochondrial pyruvate, $\operatorname{pyr}[\mathrm{m}]$ ) are considered as distinct metabolites. Thus, without considering sub-cellular compartmentalization, this model accounts for 681 unique metabolites.

In iPP668, we have included a biomass equation which is composed of biosynthetic precursors and energy requirement. Appropriate coefficients of amino acids and carbohydrates were obtained from a recent experimental composition analysis of P. pastoris [33] while lipid and sterol compositions were evaluated based on an analytical study of the cellular membranes [34]. The reported $\mathrm{G}+\mathrm{C}$ content of $41.1 \%$ [35] forms the basis of our calculation of nucleotide composition and the composition of the individual RNA is assumed to be similar to that of $\mathrm{S}$. cerevisiae [36] according to findings by [33]. We also calculated the growth associated ATP requirement for the polymerization of proteins, DNA and RNA and included this energy requirement as a part of the biomass synthesis equation (see Additional file 2 for details). 


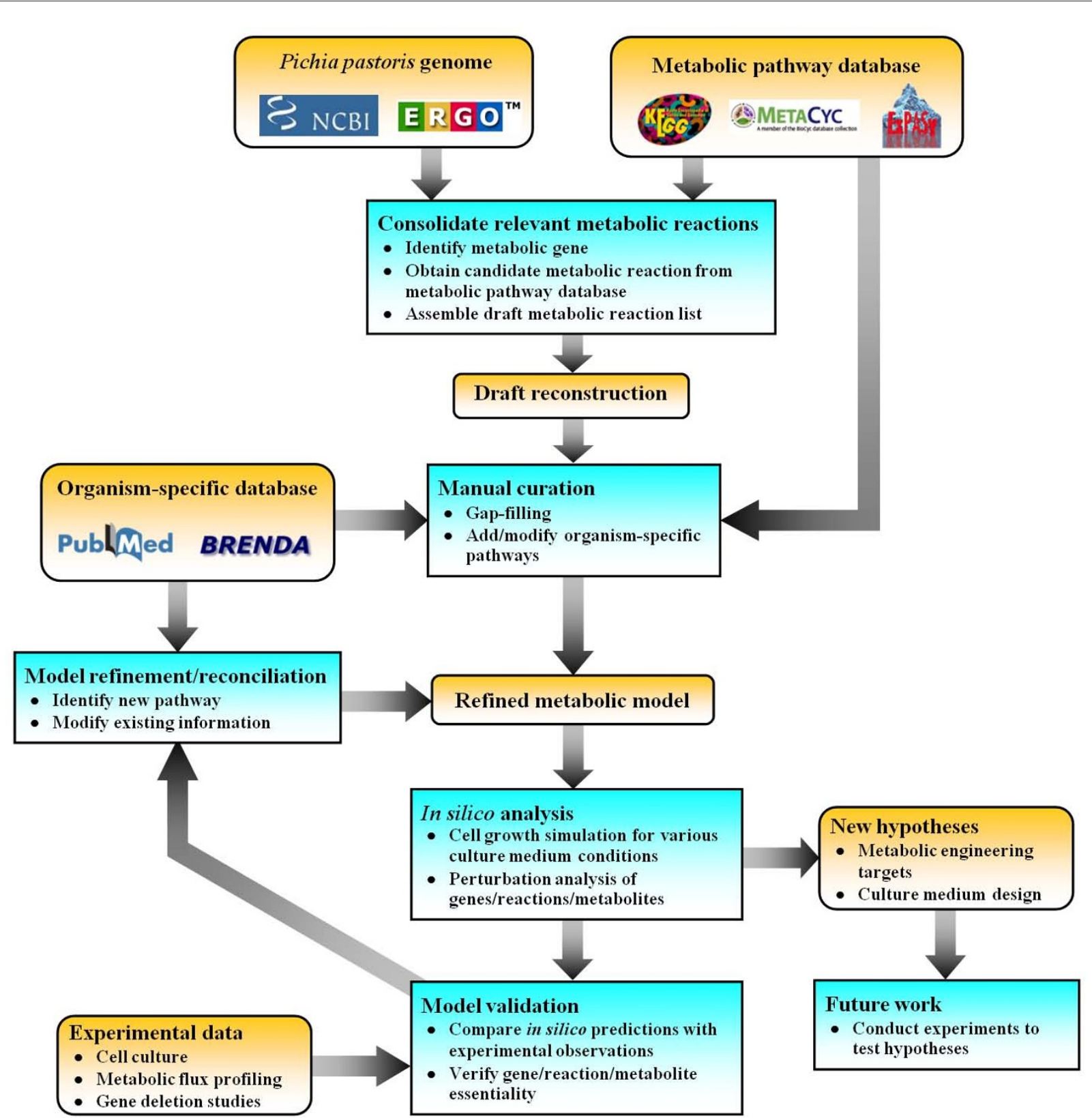

Figure 1 Reconstruction of Pichia pastoris. A recently published genome of P. pastoris [35] and various online databases, including KEGG [71], MetaCyc [70], PubMed http://www.ncbi.nlm.nih.gov/pubmed/, BRENDA [68] and ExPASy ENZYME database [69], were used to reconstruct and manually curate the iPP668 metabolic model.

Unique and conserved features in $P$. pastoris metabolism were further elucidated by comparing $i P P 668$ with two model organisms: S. cerevisiae (iMM904) [37] and E. coli (iAF1260) [25]. It should be noticed that we disregarded the sub-cellular compartmentalization of reactions for the comparative analysis of metabolic capability by eliminating inter-compartmental transport and metabolic reaction duplicates in different compartments. From this comparison, there are 292 reactions and 439 metabolites that are common to the three species (Figure 2 ). These reactions largely belong to the central carbon metabolism and amino acid biosynthetic pathways. The 415 reactions and 196 metabolites, shared only by P. pastoris and S. cerevisiae, are generally classified under the lipid and carbohydrate biosynthetic pathways while the 79 reactions and 46 metabolites unique to $P$. pastoris are mainly from the methanol utilization pathway (Figure 3) and certain parts of lipid metabolism which are yeast- 
Table 1: Summary statistics of metabolic reconstruction for two yeast models

\begin{tabular}{|c|c|c|c|c|}
\hline Genome characteristics & \multicolumn{2}{|c|}{ P. pastoris } & \multicolumn{2}{|c|}{ S. cerevisiae } \\
\hline Genome length & \multicolumn{2}{|c|}{$9.3 \mathrm{Mbp}$} & \multicolumn{2}{|c|}{ 12.1 Mbp } \\
\hline $\mathrm{G}+\mathrm{C}$ content & \multicolumn{2}{|c|}{$41.1 \%$} & \multicolumn{2}{|c|}{$38.3 \%$} \\
\hline Coding genes & \multicolumn{2}{|c|}{5,313} & \multicolumn{2}{|c|}{6,607} \\
\hline \multicolumn{5}{|l|}{ Model characteristics } \\
\hline No. of genes & \multicolumn{2}{|c|}{668} & \multicolumn{2}{|c|}{904} \\
\hline $\begin{array}{l}\text { No. of gene-associated } \\
\text { reactions }\end{array}$ & \multicolumn{2}{|c|}{1,007} & \multicolumn{2}{|c|}{1,043} \\
\hline No. of other reactions & \multicolumn{2}{|c|}{354} & \multicolumn{2}{|c|}{534} \\
\hline No. of internal reactions & \multicolumn{2}{|c|}{1,212} & \multicolumn{2}{|c|}{1,413} \\
\hline No. of exchange reactions & \multicolumn{2}{|c|}{149} & \multicolumn{2}{|c|}{164} \\
\hline No. of metabolites & \multicolumn{2}{|c|}{1,177} & \multicolumn{2}{|c|}{1,228} \\
\hline Compartment & No. of metabolites & No. of reactions & No. of metabolites & No. of reactions \\
\hline Cytosol & 607 & 623 & 634 & 709 \\
\hline Endoplasmic reticulum & 28 & 15 & 28 & 15 \\
\hline Extracellular matrix & 149 & 12 & 164 & 14 \\
\hline Golgi apparatus & 15 & 4 & 17 & 6 \\
\hline Mitochondria & 235 & 163 & 241 & 175 \\
\hline Nucleus & 35 & 16 & 40 & 16 \\
\hline Peroxisome & 84 & 66 & 80 & 65 \\
\hline Vacuole & 24 & 3 & 24 & 3 \\
\hline Inter-compartment & - & 452 & - & 574 \\
\hline Total & 1,177 & 1,361 & 1,228 & 1,577 \\
\hline
\end{tabular}

Data of S. cerevisiae is obtained from [37] and Saccharomyces Genome Database http://www.yeastgenome.org/.

specific. It is further observed that the lipid biosynthetic pathways of both yeasts are structurally identical, only differing in the composition of fatty acid chains.

\section{Validation of phenotypic predictions}

In this study, we validated the $i P P 668$ metabolic model by checking the difference between predicted cellular phenotypes and experimental observations obtained from two sets of chemostat culture conditions, glucose minimal medium (see Methods) and glycerol/methanol mixed medium [38]. In both cases, we maximized cell growth while constraining carbon source uptake rates at experimentally determined levels.

In the case of glucose minimal medium, we observed that the predicted cell growth, oxygen uptake and carbon dioxide evolution rates are highly consistent with the data obtained from our chemostat experiments (Figure 4). Discrepancies between experimental observations and model predictions are within $20 \%$ which can be consid- ered acceptable. The constantly higher growth and gaseous exchange rate predictions can be due to possible errors in biomass composition data obtained from various sources (see Methods and Additional file 2). It should be noted that in order to mimic physiological states, we specified conditional parameters (see Methods). NGAM requirement $(2.3 \mathrm{mmol} \mathrm{ATP} / \mathrm{gDCW}-\mathrm{hr})$ was estimated from our chemostat data whereas we defined $\mathrm{P} / \mathrm{O}$ ratio $(1.48 \mathrm{~mol} \mathrm{ATP} / \mathrm{mol} \mathrm{O})$ based on previous studies $[39,40]$.

Another set of chemostat culture data [38] also allowed us to evaluate the fidelity of physiological predictions under the glycerol/methanol mixed media condition. In this case, the NGAM requirement is estimated to be about $6 \mathrm{mmol}$ ATP/gDCW-hr while the $\mathrm{P} / \mathrm{O}$ ratio remains unchanged. Predictions for cell growth in glycerol minimal media are reasonably consistent with experimental observations but the discrepancy becomes significant when methanol uptake rate is high (Table 3). In the latter case, methanol uptake induces the synthesis 
Table 2: Functional classification of metabolic reactions

\begin{tabular}{|c|c|c|c|}
\hline Alanine and Aspartate Metabolism & 9 & Other Amino Acid Metabolism & 10 \\
\hline Alternate Carbon Metabolism & 21 & Oxidative Phosphorylation & 18 \\
\hline Anaplerotic reactions & 10 & Pantothenate and CoA Biosynthesis & 16 \\
\hline Arabinose Metabolism & 3 & Pentose Phosphate Pathway & 13 \\
\hline Arginine and Proline Metabolism & 31 & Phospholipid Biosynthesis & 42 \\
\hline Asparagine metabolism & 2 & Phospholipid Metabolism & 8 \\
\hline ATP maintenance & 1 & Porphyrin and Chlorophyll Metabolism & 13 \\
\hline Biomass requirement & 1 & Purine and Pyrimidine Biosynthesis & 51 \\
\hline Citric Acid Cycle & 13 & Pyridoxine Metabolism & 8 \\
\hline Complex Alcohol Metabolism & 22 & Pyruvate Metabolism & 17 \\
\hline Cysteine Metabolism & 7 & Quinone Biosynthesis & 17 \\
\hline Fatty Acid Biosynthesis & 61 & Riboflavin Metabolism & 11 \\
\hline Fatty Acid Degradation & 42 & Sphingolipid Metabolism & 57 \\
\hline Fatty Acid Metabolism & 3 & Starch and Sucrose Metabolism & 2 \\
\hline Folate Metabolism & 23 & Sterol Metabolism & 48 \\
\hline Fructose and Mannose Metabolism & 8 & Taurine Metabolism & 1 \\
\hline Galactose metabolism & 2 & Thiamine Metabolism & 12 \\
\hline Glutamate metabolism & 15 & Threonine and Lysine Metabolism & 16 \\
\hline Glutamine Metabolism & 3 & Transport, Endoplasmic Reticular & 8 \\
\hline Glycerolipid Metabolism & 11 & Transport, Extracellular & 145 \\
\hline Glycine and Serine Metabolism & 19 & Transport, Golgi Apparatus & 2 \\
\hline Glycolysis/Gluconeogenesis & 22 & Transport, Mitochondrial & 91 \\
\hline Glycoprotein Metabolism & 5 & Transport, Nuclear & 5 \\
\hline Histidine Metabolism & 14 & Transport, Peroxisomal & 20 \\
\hline Methanol Metabolism & 7 & Transport, Vacuolar & 25 \\
\hline Methionine Metabolism & 18 & tRNA charging & 35 \\
\hline NAD Biosynthesis & 18 & Tyrosine, Tryptophan, and Phenylalanine Metabolism & 34 \\
\hline Nitrogen Metabolism & 3 & Valine, Leucine, and Isoleucine Metabolism & 19 \\
\hline Nucleotide Salvage Pathway & 61 & Xylose Metabolism & 2 \\
\hline \multirow[t]{2}{*}{ Other } & 11 & Exchange reactions & 149 \\
\hline & & Total & 1361 \\
\hline
\end{tabular}

The table shows the number of reactions classified under each subsystem.

of alcohol oxidase enzyme and generation of peroxisome lipid membrane which may cause substantial alteration in the cellular composition of amino acids and lipids $[7,41]$. Moreover, the effects of metabolic regulation, such as the repression of $A O X$ by glycerol [42], that are not captured in the current model may also contribute to the discrepancy. Thus, future works in the experimental analysis of changes in biomass composition during methanol induction can provide necessary information to improve prediction capability of the current metabolic model.

\section{Carbon sources for recombinant protein production}

Among the various carbon sources available, methanol is the inducer for $A O X$ promoter which is commonly used for heterologous protein production in P. pastoris. However, the yeast typically grows very slowly on this carbon source [7]. Thus, an alternative substrate can be supplied during the initial phase to achieve high cell density, followed by shifting into methanol induction phase for protein production [43]. Based on this feeding strategy, desirable carbon sources must be able to yield high growth rate with high capacity to synthesize amino acids in the earlier phase so that available amino acid pools can be diverted to produce recombinant protein in the later phase. In this sense, we can explore the effect of various carbon sources (alanine, glucose, glycerol, methanol, sorbitol and trehalose) on cellular metabolism of P. pastoris using the reconstructed model and as such, identify the 

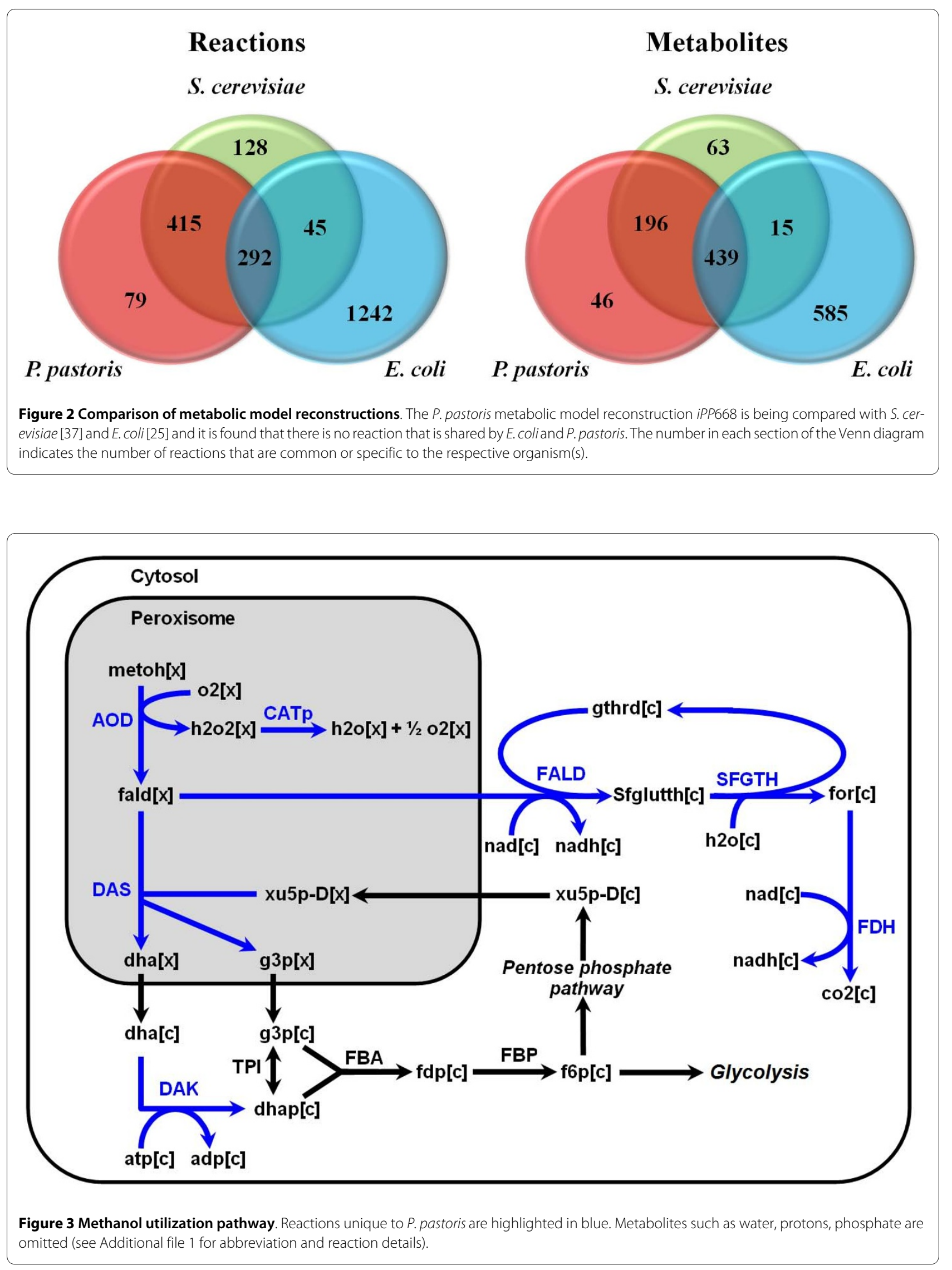

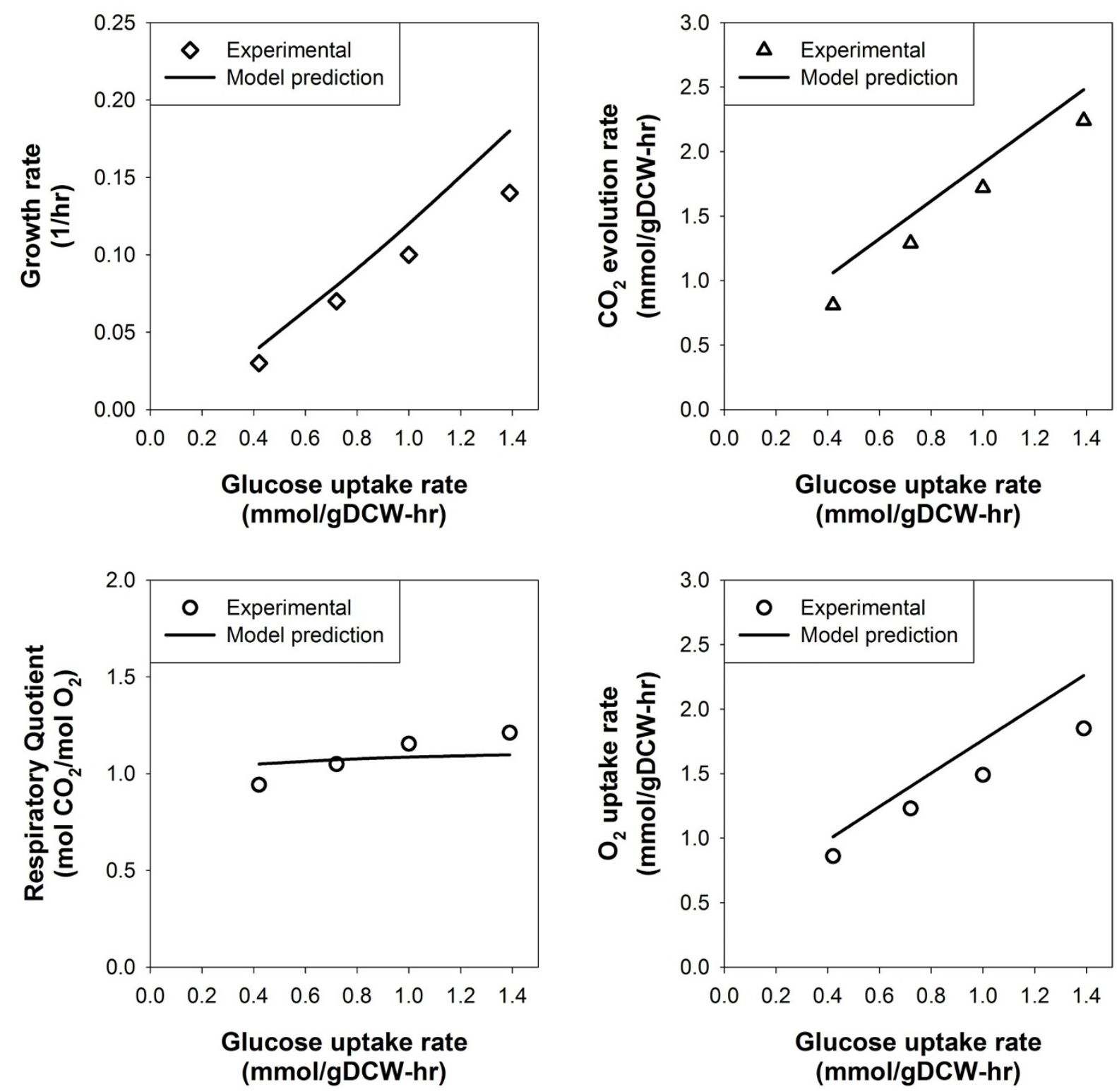

Figure 4 Chemostat culture simulation results. Data points for each chemostat experimental data point were generated by constraints-based flux analysis implemented with glucose uptake rate constrained at the respective experimentally determined values.

best candidate for recombinant protein production. To do so, we simulated cell growth by constraining the supply of each carbon source to one C-mmol/gDCW-hr (i.e. $0.167 \mathrm{mmol} / \mathrm{gDCW}$-hr for glucose, $0.333 \mathrm{mmol} / \mathrm{gDCW}$ hr for glycerol, $1 \mathrm{mmol} / \mathrm{gDCW}$-hr for methanol, etc.).

Based on the first criterion of high growth yield, glycerol is the most promising candidate for recombinant protein production, followed by sorbitol (Figure 5). Generally, high utilization of the central metabolism can lead to increased production of various precursors, which can further synthesize building blocks required for the bio- mass. However, the resulting flux distributions indicated that the utilization of central carbon metabolism is the highest for methanol uptake despite yielding the lowest growth rate. We can understand this observation by examining gaseous exchange rates and ATP flux-sum for methanol utilization. The significantly higher turnover rate of ATP and gaseous exchange rates suggest that much of the resources have been diverted to energy generation during methanol utilization. The low respiratory quotient resulting from this diversion of resources is consistent with findings from several experimental studies 


\begin{tabular}{|c|c|c|c|c|c|c|c|c|}
\hline & \multicolumn{8}{|c|}{ Dilution rate, $D$} \\
\hline & \multicolumn{2}{|c|}{$D=0.16$} & \multicolumn{2}{|c|}{$D=0.16$} & \multicolumn{2}{|c|}{$D=0.05$} & \multicolumn{2}{|c|}{$D=0.05$} \\
\hline & $\operatorname{Exp}^{a}$ & Pred & $\operatorname{Exp}^{a}$ & Pred & $\operatorname{Exp}^{a}$ & Pred & $\operatorname{Exp}^{a}$ & Pred \\
\hline Cell growth & 0.16 & 0.18 & 0.16 & 0.19 & 0.05 & 0.05 & 0.05 & 0.05 \\
\hline Glycerol uptake ${ }^{b}$ & \multicolumn{2}{|c|}{2.75} & \multicolumn{2}{|c|}{2.23} & \multicolumn{2}{|c|}{1.09} & \multicolumn{2}{|c|}{0.57} \\
\hline Methanol uptake & \multicolumn{2}{|c|}{-} & \multicolumn{2}{|c|}{2.73} & \multicolumn{2}{|c|}{-} & \multicolumn{2}{|c|}{2.33} \\
\hline $\mathrm{CO}_{2}$ evolution & 2.35 & 2.40 & 3.60 & 3.22 & 1.56 & 1.72 & 2.21 & 2.37 \\
\hline $\mathrm{O}_{2}$ uptake & 3.62 & 3.56 & 7.20 & 5.47 & 2.16 & 2.20 & 4.85 & 3.76 \\
\hline Respiratory quotient & 0.65 & 0.68 & 0.50 & 0.59 & 0.72 & 0.78 & 0.46 & 0.63 \\
\hline $\mathrm{P} / \mathrm{O}$ ratio & - & 1.48 & - & 1.48 & - & 1.47 & - & 1.49 \\
\hline
\end{tabular}

In the chemostat culture, growth rate is equivalent to dilution rate. Units of measurements are given as follows: growth/dilution rate, 1/hr; carbon source uptake or gaseous exchange rates, $\mathrm{mmol} / \mathrm{gDCW}$ - $\mathrm{hr}$; Respiratory quotient, $\mathrm{mol} \mathrm{CO}_{2} / \mathrm{mol} \mathrm{O}_{2} ; \mathrm{P} / \mathrm{O}$ ratio, $\mathrm{mol}$ ATP $/ \mathrm{mol} \mathrm{O}$.

a Data from [38].

${ }^{b}$ Carbon source uptake rates are model input parameters which are not predicted.

$[38,44]$. Hence, the higher utilization of central metabolism is a consequence of the high energy requirement of methanol metabolism in P. pastors.

Not surprisingly, flux-sum distribution profiles on various carbon sources confirmed that sorbitol and glycerol are capable of generating higher amino acid flux-sum compared to others. Thus, this large pool of amino acids can potentially be diverted to synthesize recombinant protein during the induction phase, indicating sorbitol and glycerol are desirable carbon sources for recombinant protein production in terms of both growth yield and amino acid biosynthetic capability (Figure 5). However, if $A O X$ promoter is used for recombinant protein expression, sorbitol will be more superior as glycerol is known to repress the promoter [42]. Therefore, the current model-driven evaluation of various carbon sources recommends a feeding strategy of supplying sorbitol as the co-substrate during methanol induction phase to provide the necessary resources for recombinant protein production.

\section{Application of $P$. pastoris for whole-cell biotransformation}

Apart from recombinant protein production through methanol induction, we managed to identify another potential application of culturing P. pastoris in methanol. From the in silico analysis, it was observed that growing $P$. pastoris in methanol minimal media can exhibit high turnover of NADH, implying the potential capability of $P$. pastoris for whole-cell biotransformation. For example, value-added chemical precursors, e.g. 2,3-butanediol, can be synthesized via enzymatic reduction of ketones, e.g. acetoin, which requires NADH or NADPH regeneration [45-49]. In this aspect, the high rate of NADH regeneration in P. pastoris is suitable for biocatalysis of butanediol dehydrogenase reaction to convert acetoin into 2,3butanediol [50]. To achieve cost-effectiveness, we can further enhance the NADH regeneration of $P$. pastoris by identifying gene targets to be overexpressed through the modified flux analysis implementation (see Methods).

Simulation results show that when $P$. pastoris is supplied with $1 \mathrm{mmol} / \mathrm{gDCW}-\mathrm{hr}$ of acetoin, attenuating the flux activity of some reactions can give rise to a proportional decrease in the maximum achievable butanediol dehydrogenase reaction flux (Figure 6). Fixing the fluxes of these reactions imposes an upper limit on the flux of butanediol dehydrogenase. Thus, such reactions are "directionally coupled" to 2,3-butanediol production, according to [51]. For the in vivo system, the directionally coupled reactions will become bottlenecks only if the corresponding enzymes were not expressed adequately while the maximum rate of 2,3-butanediol production may be achievable upon the expression of the enzymes at high levels. From this inference, the list of target enzymes identified can be genetically enhanced for relieving the bottleneck if the experimentally observed 2,3-butanediol production is significantly less than the theoretical value.

\section{Discussion}

The genome-scale metabolic modeling and analysis procedure presented in this study can potentially be used to design culture media for enhancing the performance of $P$. pastoris. We have shown through the analysis of carbon source utilization that glycerol and sorbitol are the best candidates for achieving high growth yields in P. pastoris cell culture (Figure 5). Furthermore, with regards to recombinant protein production using the $A O X$ promoter, sorbitol is clearly better than glycerol since the latter represses the $A O X$ promoter [42]. The generation of 

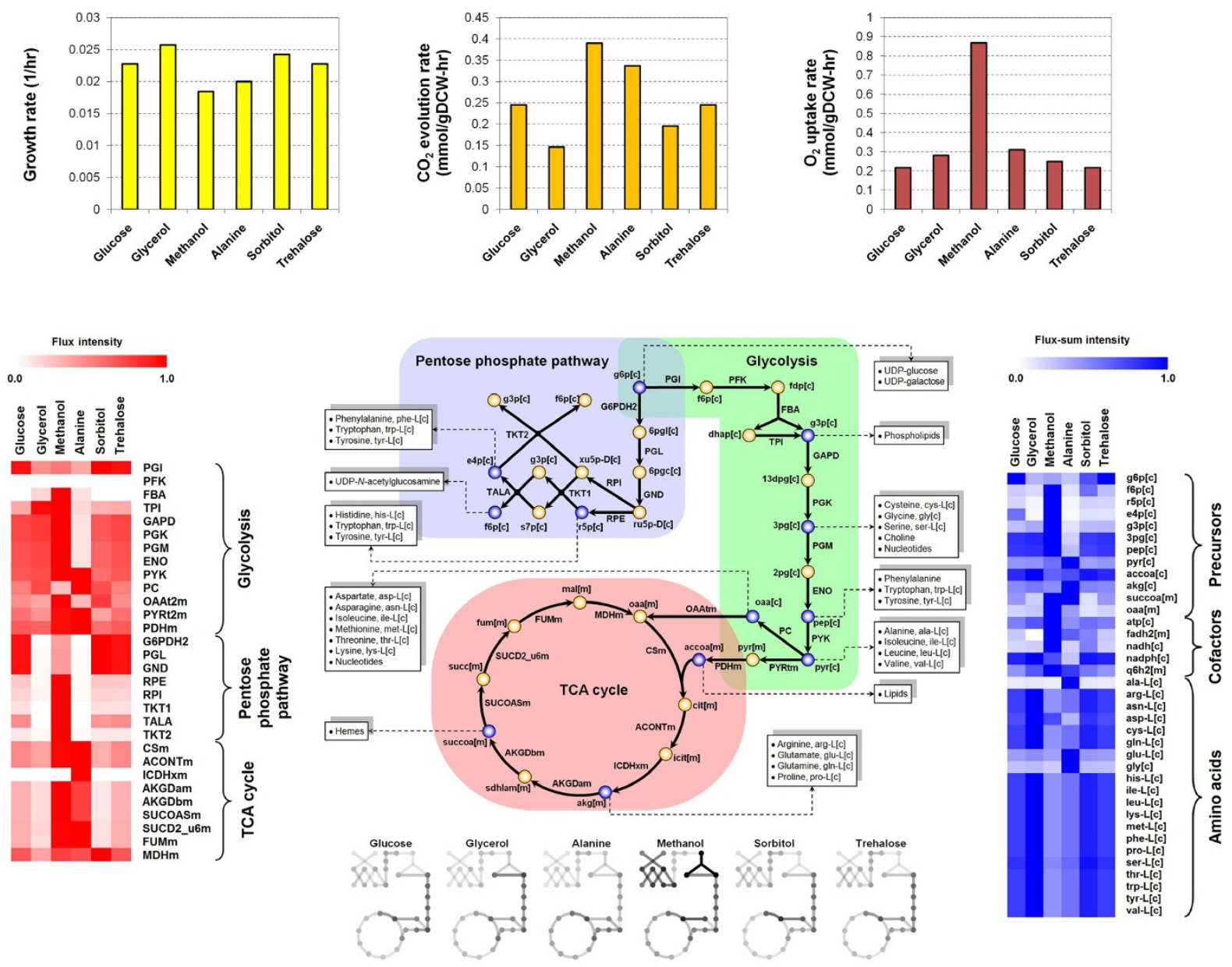

Figure 5 Flux and flux-sum distributions for different carbon sources. Growth rate, flux and flux-sum values were generated based on individual carbon source uptake rate of $1 \mathrm{C}-\mathrm{mmol} / \mathrm{gDCW}$-hr. The color intensity of the lines in the central carbon metabolic network corresponds to the flux values. Precursor metabolites are in blue and the building blocks derived from each of them are specified in the text boxes. The heat-maps on the left and right illustrate the flux and flux-sum distributions, respectively. Similarly, the color intensity corresponds to the flux or flux-sum values normalized with respect to the maximum for each reaction or metabolite.

high flux-sum for many of the amino acids due to sorbitol utilization (Figure 5) can provide a possible explanation for the successful use of sorbitol in recombinant protein expression experiments which utilizes methanol induction [52-55]. Although the technique of expressing recombinant protein using the $A O X$ promoter has been successful, alternative promoters, such as gylceraldehyde 3-phosphate dehydrogenase (GAP), glutathione-dependent formaldehyde dehydrogenase (FLD1), peroxisomal matrix protein (PEX8) and secretion GTPase (YPT1) promoters, have been proposed since methanol, a petroleum compound, may not be perceived as an appropriate raw material for production of proteins for human consumption [56]. For the utilization of these alternative promoters, sorbitol may not be the best candidate due to regulatory factors and further analysis using $i P P 668$ can provide us with the corresponding best carbon source selection.

Another application of $i P P 668$ is the rational identification of metabolic engineering targets. From our analysis of carbon source utilization, we found that growing $P$. pastoris in methanol minimal medium is suitable for biocatalysis of ketone reduction such as the conversion of acetoin into 2,3-butanediol, which requires NADH regeneration [50]. In this case, the key enzyme, butanediol dehydrogenase, is overexpressed but the native metabolism of the host may consist of some limiting reactions or bottlenecks that prevent the maximum utilization of the butanediol dehydrogenase reaction. Some of the bottleneck reactions, AOD, FALD and FDH, identi- 


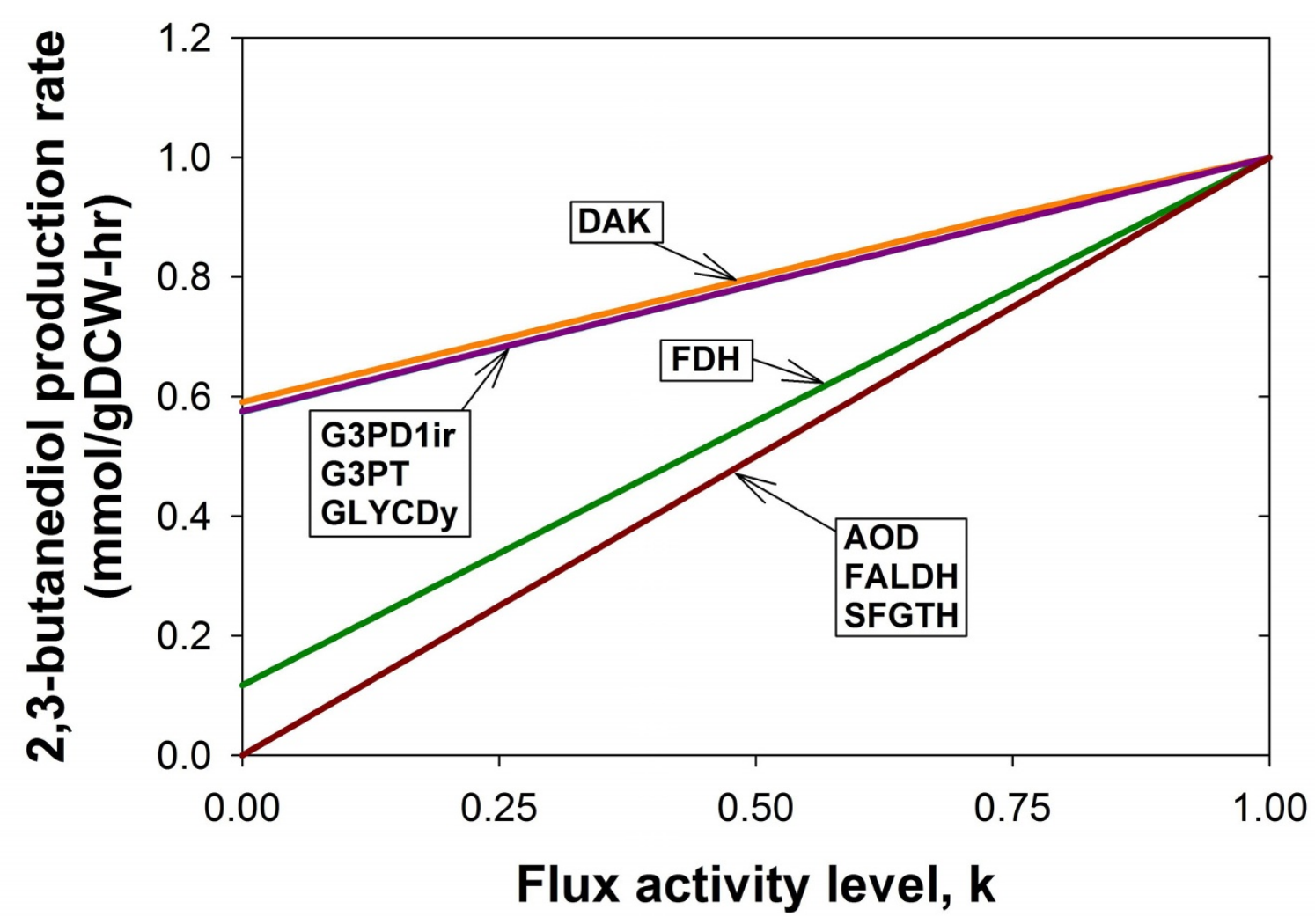

Figure 6 Effect of flux activity on 2,3-butanediol production. The flux activity level shown on the $x$-axis refers to the flux activity normalized with respect to the maximum flux activity value for each reaction. This value is also equivalent to the factor k used to carry out the analysis (see Methods). Reaction abbreviations: AOD, alcohol oxidase; DAK, dihydroxyacetone kinase; FALDH, formaldehyde dehydrogenase; FDH, formate dehydrogenase; G3PD1 ir, glycerol-3-phosphate dehydrogenase; G3PT, glycerol-3-phosphatase; GLYCDy, glycerol dehydrogenase; SFGTH, S-formylglutathione hydralase (see Additional file 1 for details).

fied by our in silico analysis have been demonstrated to be appropriate metabolic engineering targets for enhancing 2,3-butanediol production [50] and the other potential targets, DAK, G3PD1ir, G3PT and GLYCDy, can serve as hypotheses for future experimental validation. Hence, using the proposed computational framework, iPP668 can be a useful tool for tackling other metabolic engineering problems associated with $P$. pastoris.

Protein glycosylation is an important post-translational process which can affect the protein's secretion, folding and bioactivity $[57,58]$. Thus the extension of $i P P 668$ to account for glycosylation processes can potentially increase the utility of the model. The design of glycosylation pathway through glycoengineering is critical for recombinant protein production in yeasts [3]. When combined with synthetic biology, effective glycoengineering techniques can even produce synthetic glycoproteins with enhanced therapeutic function [59]. Hence, it is highly desirable to develop a framework to rationally design the glycosylation pathway by analyzing the interaction between cellular metabolism and post-translational modification mechanisms, and subsequently optimizing the production of desired glycoforms, as shown in previous theoretical studies [60-63]. Although the limitations of constraints-based flux analysis prohibit detail modeling of the diverse range of glycoforms, it is still possible to account for the overall metabolic requirement involved in protein glycosylation as discussed by [26]. We can characterize the glycan structure of any target glycoprotein and evaluate the stoichiometry of monosaccharides required to synthesize it using advanced glycomics techniques [64]. We can then construct a lumped glycoprotein synthesis equation that also describes the energy requirement of glycosidic bond formation [65]. By adding this equation to the existing metabolic model, we can investigate the sensitivity of cellular metabolism to the carbohydrate and energy requirements of glycosylation. Consequently, this extended model offers a good platform for the integration of glycomics and other omics, such as metabolomics and fluxomics, for a large-scale systems analysis of the cellular physiology in Pichia pastoris.

During the peer review process of the current work, we identified another independent reconstruction effort that 
has been made by Sohn et al. [66], presenting the genome-scale model of $P$. pastoris metabolism, PpaMBEL1254. A model comparison between iPP668 and PpaMBEL1254 shows that both models are fully compartmentalized into 8 subcellular compartments, and capable of describing P. pastoris cellular metabolism under various carbon source uptake conditions. Nonetheless, several differences between the current study and Sohn et al. can be found with respect to the model size, validation and subsequent analysis. The size of iPP668 (668 genes, 1,361 reactions, 1,177 metabolites) is larger, providing more information than PpaMBEL1254 (540 genes, 1,254 reactions, 1,147 metabolites). In addition, iPP668 was validated by two sets of chemostat experimental data while Sohn et al. used batch fermentation data for qualitative model validation. Interestingly, Sohn et al. explored the capability of $P$. pastoris to produce recombinant proteins such as human serum albumin and human superoxide dismutase under various oxygenation rates using PpaMBEL1254, while we have discussed the general procedure to represent the protein production in the model based on amino acid biosynthetic rates. However, the PpaMBEL1254 model did not clearly describe the methanol utilization pathway of $P$. pastoris which is an important metabolic characteristic of great interest for applications in recombinant protein production and biotransformation, as discussed in the current study. Therefore, we suggest the combination of iPP668 and PpaMBEL1254 for future model expansion and systems metabolic engineering studies to harness the useful information provided by two genome-scale metabolic models.

\section{Conclusion}

In this study, we have reconstructed a genome-scale metabolic model of the methylotrophic yeast $P$. pastoris, which has been developed for recombinant protein production as well as whole-cell biotransformation. The metabolic model was manually curated with information from literature and various databases to provide a good description of cell growth under various culture conditions through the use of constraints-based flux analysis. Validation of the curated model has shown that the model was able to reproduce key characteristics of $P$. pastoris metabolism reported in various experimental studies. The analysis of $P$. pastoris growth on different substrates has also allowed us to understand some useful application of the methylotrophic yeast and to generate testable hypotheses which can help to improve its biochemical production capability. Future works in the experimental analysis of $P$. pastoris can potentially enhance the predictive capability of the current model which can be integrated with high-throughput omics analysis for yeast systems biotechnology.

\section{Methods}

\section{Reconstruction of metabolic model}

The metabolic network of $P$. pastoris was reconstructed based on a recently published genome annotation of the organism [35]. An initial draft of the network is constructed by compiling annotated metabolic genes; relevant reactions are added with the respective geneprotein-reaction (GPR) assignments [23] based on information from online databases such as BioSilico [67], BRENDA [68], ExPASy ENZYME [69], MetaCyc [70] and KEGG [71] . The initial draft is subsequently refined through manual curation and gap-filling processes. In addition, constraints-based flux analysis was used to identify missing links that can be filled by adding necessary steps leading to the cell growth. The process of model refinement typically requires additional information from scientific references and publications which can be found in the PubMed database. Subsequently, relevant experimental validation and model analysis further refine the model in an iterative manner (Figure 1).

\section{Constraints-based flux analysis}

Cellular metabolism can be simulated by constraintsbased flux analysis as described elsewhere [72-74]. We used this approach to evaluate cell growth of $P$. pastoris under various culture conditions. Mathematically, the rate of biomass synthesis forms the objective function to be maximized, subjected to stoichiometric, thermodynamic and reaction capacity constraints, resulting in the following linear programming problem:

$$
\begin{aligned}
& \max v_{\text {biomass }} \\
& \text { Subject to : } \\
& \qquad \sum_{j} S_{i j} v_{j}=0 \\
& \alpha_{j} \leq v_{j} \leq \beta_{j}
\end{aligned}
$$

where $S_{i j}$ refers to the stoichiometric coefficient of metabolite $i$ involved in reaction $j$ and $v_{j}$ is the flux of reaction $j$. Reaction capacity and thermodynamic constraints, based on reaction reversibility information, are specified using the parameters $\alpha_{j}$ and $\beta_{j}$ indicating the lower and upper bounds of reaction $j$, respectively. Unless experimental measurements are available, we typically specify $\beta_{j}=\inf$ and $\alpha_{j}=-\inf$ (for reversible reactions) or $\alpha_{j}$ $=0$ (for irreversible reactions). In addition, gene deletion can be simulated by setting $\alpha_{j}=\beta_{j}=0$ for the associated metabolic reactions. In this study, the linear programming problem was solved using General Algebraic Modeling System (GAMS) Integrated Development Environment (IDE) version 22.7 [75] 


\section{Stipulation of condition-specific parameters}

The values of condition-specific parameters, such as nongrowth associated ATP maintenance requirement (NGAM) and P/O ratio, vary with culture media and other environmental conditions. Thus, these values have to be determined for simulation of different experimental conditions. NGAM requirement is specified by fixing the flux value of ATP maintenance reaction. The experimental value is determined by plotting the uptake rates of carbon source against dilution rates from a chemostat run (see Additional file 2 for calculation details). $\mathrm{P} / \mathrm{O}$ ratio is defined as the ratio of ATP produced to oxygen atom reduced by the respiratory chain. Thus, this value can be determined by calculating $0.5\left(v_{\mathrm{ATPS} 3 \mathrm{~m}} / v_{\mathrm{CYOOm}}\right)$ where ATPS3m and CYOOm refer to ATP synthase and cytochrome c oxidase reactions in mitochondria compartment, respectively. It can be changed by adjusting stoichiometric coefficients of hydrogen ions in the cytochrome c oxidase reaction. (See Additional file 1 for reaction details.)

\section{Describing metabolite turnover rate using flux-sum}

The metabolic state of a cellular system can be described by the interconversion of metabolites. Here, the turnover rate of intermediate metabolites is defined as their fluxsum $[76,77]$. Since the overall consumption and generation rates are equal under the steady-state assumption, the flux-sum of metabolite $i$ can be formulated as $0.5 \sum_{j}\left|S_{i j} v_{j}\right|$. Each $\left|S_{i j} v_{j}\right|$ term in this summation series gives us the absolute rate of consumption/generation of metabolite $i$ due to reaction $j$ and thus by halving the sum of these terms, we can obtain the overall turnover rate for metabolite $i$.

\section{Identification of gene targets for overexpression}

In metabolic engineering, bottlenecks in the metabolic network can be considered as potential gene targets to be overexpressed [78]. Therefore, it is important to devise a systematic framework for identifying such targets. In this regard, we modified the flux analysis procedure to detect relevant reactions that can potentially affect the biosynthetic rate of the product of interest $\left(v_{\text {target }}\right)$. In our computational framework, $v_{\text {target }}$ is maximized under different levels of reaction flux perturbation. Taking into consideration that the enhancement of biochemical production typically results in attenuated growth rate, we set the lower limit of cell growth to $50 \%$ of the maximum value $\left(v_{\text {biomass }}^{\max }\right)$. The mathematical formulation can lead to the following mixed integer linear programming (MIP) problem:

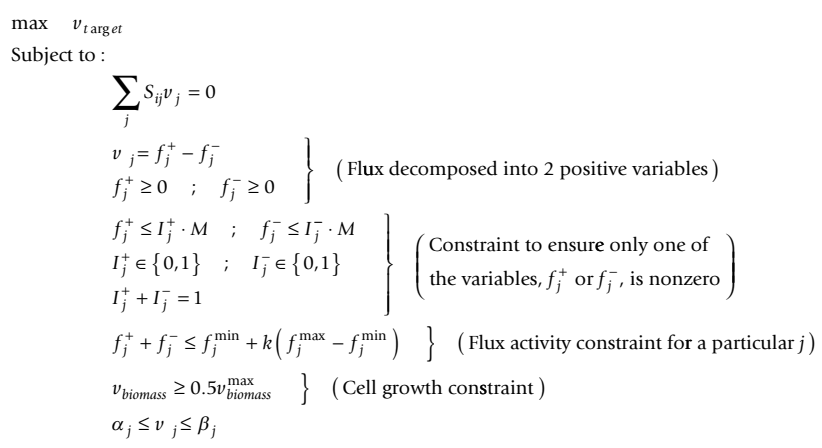

We define "flux activity" as the absolute value of a reaction flux. Thus $f_{j}^{\max }$ and $f_{j}^{\min }$ refer to the maximum and minimum flux activity of reaction $j$ respectively, under the cell growth constraint of $0.5 \cdot v_{\text {biomass }}^{\max }$. In the above formulation, big $M$ is some arbitrarily chosen large value representatiing the flux upper limit which we typically specify as $1000 \mathrm{mmol} / \mathrm{gDCW}$-hr. By solving the MIP problem for each reaction $j$ and different values of $k$ (in this case: $0,0.25,0.5,0.75$ and 1.0), we can evaluate the effect of individual reaction flux activity on the flux of any target reaction. The MIP is implemented and solved in GAMS IDE version 22.7 [75].

\section{Strain and chemostat culture}

A wild type Pichia pastoris X-33 (Invitrogen, Carlsbad, CA, USA) was used for the chemostat culture. A single colony of the strain was inoculated into $20 \mathrm{~mL}$ of yeast protein dextrose (YPD) medium containing (per liter): 20 $\mathrm{g}$ of glucose, $10 \mathrm{~g}$ of yeast extract, and $20 \mathrm{~g}$ of Bacto-peptone and incubated overnight at $30^{\circ} \mathrm{C}$. The culture was transferred to a $500 \mathrm{~mL}$ Erlenmeyer flask containing 100 $\mathrm{mL}$ of YPD broth and incubated overnight at $30^{\circ} \mathrm{C}$. This culture was used as a seed for the chemostat culture using $2.5 \mathrm{~L}$ jar fermentors (KoBiotech, Incheon, Korea) with a constant volume of $800 \mathrm{~mL}$. Cells were first batch-cultured in a defined glucose minimal medium containing (per liter): 50 g glucose, $1.5 \mathrm{~g} \mathrm{MgSO}_{4} \cdot 7 \mathrm{H}_{2} \mathrm{O}, 0.2 \mathrm{mg}$ Biotin,

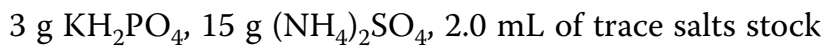
solution and $1.0 \mathrm{~mL}$ of a solution containing $2 \mathrm{~g} / \mathrm{L}$ biotin 
in $1 \mathrm{M} \mathrm{NaOH}$. The trace element solution contained (per liter) $5 \quad \mathrm{~mL} \quad \mathrm{H}_{2} \mathrm{SO}_{4}, 4.0 \mathrm{~g} \quad \mathrm{CuSO}_{4} \cdot 5 \mathrm{H}_{2} \mathrm{O}, \quad 14.0 \mathrm{~g}$ $\mathrm{MnSO}_{4} \cdot \mathrm{H}_{2} \mathrm{O}, 2.6 \mathrm{~g} \mathrm{Na}_{2} \mathrm{MoO}_{4} \cdot 2 \mathrm{H}_{2} \mathrm{O}, 4.0 \mathrm{~g} \mathrm{H}_{3} \mathrm{BO}_{3}, 4.0 \mathrm{~g}$ $\mathrm{CoCl}_{2} \cdot 6 \mathrm{H}_{2} \mathrm{O}, 22.0 \mathrm{~g} \mathrm{ZnSO}_{4} \cdot 7 \mathrm{H}_{2} \mathrm{O}, 55.0 \mathrm{~g} \mathrm{CaCl}_{2} \cdot 2 \mathrm{H}_{2} \mathrm{O}$ and $37.5 \mathrm{~g} \mathrm{FeCl}_{3} \cdot 6 \mathrm{H}_{2} \mathrm{O}$. Then, the chemostat culture was initiated after the depletion of glucose at the end of batch culture and continued at different dilution rates under the same medium condition. The cultures were carried-out at $30^{\circ} \mathrm{C}$ and $\mathrm{pH} 6.0$ controlled by $24 \% \mathrm{NH}_{4} \mathrm{OH}$. Agitation and aeration were fixed at $800 \mathrm{rpm}$ and $1 \mathrm{vvm}$, respectively, and the dissolved oxygen in the culture medium level was maintained above $10 \%$ air saturation during the entire culture period. Cultures were assumed to be in steady-state after at least 5 volumes changes.

\section{Analytical methods}

The concentration of biomass was determined as dry cell weight (DCW). Culture samples were collected after achieving steady-state for different dilution rates. The collected samples were centrifuged and washed twice with ultrapure water and then the pellet was dried at $100^{\circ} \mathrm{C}$. The culture supernatants were used for measuring the concentration of glucose, organic acids, glycerol and ethanol by high-pressure liquid chromatography (Gilson, Middleton, WI, USA) equipped with an HPX $87 \mathrm{H}$ column (Bio-Rad, Hercules, CA, USA), an ERC-7515A RI detector (ERC, Tokyo, Japan) and a UV detector (Youngin, Seoul, Korea). Analysis of carbon, nitrogen and hydrogen content was performed with a CHN analyzer (Carlo Erba Instruments, Rodano, Italy) of lyophilized cells as suggested by [79]. The ash content was determined by placing weighed quantities of lyophilized cells in ceramic crucibles, followed by incubation at $550^{\circ} \mathrm{C}$ for 6 hours. The exhaust gas leaving the fermentor was measured by LKM200A exhaust $\mathrm{O}_{2}$ and $\mathrm{CO}_{2}$ analyzer (Lokas, Daejeon, Korea). The carbon dioxide evolution rate (CER) and oxygen uptake rate (OUR) were deduced from the inlet and outlet carbon dioxide and oxygen compositions.

\section{Additional material}

Additional file 1 Excel file contains metabolic reaction, metabolite and gene lists.

Additional file $\mathbf{2}$ Word document contains details on the calculation of biomass composition, carbon balance and ATP maintenance requirement.

\section{Competing interests}

The authors declare that they have no competing interests.

\section{Authors' contributions}

BKSC performed the model reconstruction and drafted the manuscript. BKSC and SS performed the model analysis and simulations. JR, HL(1), JA and HL(2) performed the chemostat experiments. DYL coordinated and directed the project, and prepared the final manuscript. All authors have read and approved the final manuscript.

\section{Acknowledgements}

The work was supported by the Academic Research Fund (R-279-000-258-112) from the National University of Singapore and the Biomedical Research Council of A*STAR (Agency for Science, Technology and Research), Singapore.

\section{Author Details}

1 NUS Graduate School for Integrative Sciences and Engineering, National University of Singapore, 28 Medical Drive, \#05-01, 117456, Singapore, 2Bioprocessing Technology Institute, Agency for Science, Technology and Research (A*STAR), 20 Biopolis Way, \#06-01, Centros, 138668, Singapore,

${ }^{3}$ Biotechnology Process Engineering Center, KRIBB, Daejeon 305-806, Republic of Korea and 4 Department of Chemical and Biomolecular Engineering, National University of Singapore, 4 Engineering Drive 4, 117576, Singapore

Received: 17 May 2010 Accepted: 1 July 2010

Published: 1 July 2010

\section{References}

1. Durocher $Y$, Butler M: Expression systems for therapeutic glycoprotein production. Curr Opin Biotechnol 2009, 20:700-707.

2. Hamilton SR, Gerngross TU: Glycosylation engineering in yeast: the advent of fully humanized yeast. Curr Opin Biotechnol 2007, 18:387-392.

3. Chiba $\mathrm{Y}$, Akeboshi $\mathrm{H}$ : Glycan engineering and production of 'humanized' glycoprotein in yeast cells. Biol Pharm Bull 2009, 32:786-795.

4. Gerngross TU: Advances in the production of human therapeutic proteins in yeasts and filamentous fungi. Nat Biotechnol 2004 22:1409-1414.

5. Walsh G: Biopharmaceutical benchmarks 2006. Nat Biotechnol 2006 24:769-776

6. Stratton J, Chiruvolu V, Meagher M: High cell-density fermentation. Methods in Molecular Biology 1998, 103:107-120.

7. Cregg JM: Introduction: distinctions between Pichia pastoris and other expression systems. Methods Mol Biol 2007, 389:1-10.

8. Faber KN, Harder W, Ab G, Veenhuis M: Review: methylotrophic yeasts as factories for the production of foreign proteins. Yeast 1995, 11:1331-1344

9. Cregg JM, Vedvick TS, Raschke WC: Recent advances in the expression of foreign genes in Pichia pastoris. Nature Biotechnology 1993, 11:905-910.

10. Macauley-Patrick S, Fazenda ML, McNeil B, Harvey LM: Heterologous protein production using the Pichia pastoris expression system. Yeast 2005, 22:249-270

11. Martinet W, Maras M, Saelens X, Jou WM, Contreras R: Modification of the protein glycosylation pathway in the methylotrophic yeast Pichia pastoris. Biotechnology Letters 1998, 20:1171-1177.

12. Vassileva A, Chugh DA, Swaminathan S, Khanna N: Expression of hepatitis $B$ surface antigen in the methylotrophic yeast Pichia pastoris using the GAP promoter. J Biotechnol 2001, 88:21-35.

13. Rahbarizadeh F, Rasaee MJ, Forouzandeh M, Allameh AA: Over expression of anti-MUC1 single-domain antibody fragments in the yeast Pichia pastoris. Mol Immunol 2006, 43:426-435.

14. Yao XQ, Zhao HL, Xue C, Zhang W, Xiong XH, Wang ZW, Li XY, Liu ZM: Degradation of HSA-AX15(R13K) when expressed in Pichia pastoris can be reduced via the disruption of YPS1 gene in this yeast. J Biotechnol 2009, 139:131-136.

15. Lee SY, Lee DY, Kim TY: Systems biotechnology for strain improvement. Trends Biotechnol 2005, 23:349-358

16. Otero JM, Nielsen J: Industrial systems biology. Biotechnol Bioeng 2010 105:439-460

17. Nielsen J, Jewett MC: Impact of systems biology on metabolic engineering of Saccharomyces cerevisiae. FEMS Yeast Res 2008, 8:122-131.

18. Petranovic $D$, Vemuri GN: Impact of yeast systems biology on industrial biotechnology. J Biotechnol 2009, 144:204-211.

19. Bro C, Regenberg B, Forster J, Nielsen J: In silico aided metabolic engineering of Saccharomyces cerevisiae for improved bioethanol production. Metab Eng 2006, 8:102-111.

20. Hjersted JL, Henson MA, Mahadevan R: Genome-scale analysis of Saccharomyces cerevisiae metabolism and ethanol production in fedbatch culture. Biotechnol Bioeng 2007, 97:1190-1204. 
21. Gonzalez R, Andrews BA, Molitor J, Asenjo JA: Metabolic analysis of the synthesis of high levels of intracellular human SOD in Saccharomyces cerevisiae rhSOD 2060411 SGA122. Biotechnol Bioeng 2003, 82:152-169.

22. Graf A, Dragosits M, Gasser B, Mattanovich D: Yeast systems biotechnology for the production of heterologous proteins. FEMS Yeast Res 2009, 9:335-348.

23. Feist AM, Herrgard MJ, Thiele I, Reed JL, Palsson BO: Reconstruction of biochemical networks in microorganisms. Nat Rev Microbiol 2009, 7:129-143.

24. Oberhardt MA, Palsson BO, Papin JA: Applications of genome-scale metabolic reconstructions. Mol Syst Biol 2009, 5:320.

25. Feist AM, Henry CS, Reed JL, Krummenacker M, Joyce AR, Karp PD, Broadbelt LJ, Hatzimanikatis V, Palsson BO: A genome-scale metabolic reconstruction for Escherichia coli K-12 MG1655 that accounts for 1260 ORFs and thermodynamic information. Mol Syst Bio/ 2007, 3:121.

26. Selvarasu S, Karimi IA, Ghim GH, Lee DY: Genome-scale modeling and in silico analysis of mouse cell metabolic network. Mol Biosyst 2010, 6:152-161.

27. Duarte NC, Becker SA, Jamshidi N, Thiele I, Mo ML, Vo TD, Srivas R, Palsson BO: Global reconstruction of the human metabolic network based on genomic and bibliomic data. Proc Natl Acad Sci USA 2007, 104:1777-1782

28. Fong SS, Palsson BO: Metabolic gene-deletion strains of Escherichia coli evolve to computationally predicted growth phenotypes. Nat Genet 2004, 36:1056-1058

29. Lee KH, Park JH, Kim TY, Kim HU, Lee SY: Systems metabolic engineering of Escherichia coli for L-threonine production. Mol Syst Biol 2007, 3:149.

30. Lee SJ, Lee DY, Kim TY, Kim BH, Lee J, Lee SY: Metabolic engineering of Escherichia coli for enhanced production of succinic acid, based on genome comparison and in silico gene knockout simulation. Appl Environ Microbiol 2005, 71:7880-7887.

31. Backlund PS Jr, Chang CP, Smith RA: Identification of 2-keto-4methylthiobutyrate as an intermediate compound in methionine synthesis from 5'-methylthioadenosine. J Biol Chem 1982, 257:4196-4202

32. Hartner FS, Glieder A: Regulation of methanol utilisation pathway genes in yeasts. Microb Cell Fact 2006, 5:39

33. Carnicer M, Baumann K, Toplitz I, Sanchez-Ferrando F, Mattanovich D, Ferrer $\mathrm{P}$, Albiol J: Macromolecular and elemental composition analysis and extracellular metabolite balances of Pichia pastoris growing at different oxygen levels. Microb Cell Fact 2009, 8:65

34. Wriessnegger T, Leitner E, Belegratis MR, Ingolic E, Daum G: Lipid analysis of mitochondrial membranes from the yeast Pichia pastoris. Biochim Biophys Acta 2009, 1791:166-172.

35. De Schutter K, Lin YC, Tiels P, Van Hecke A, Glinka S, Weber-Lehmann J, Rouze $P$, Van de Peer $Y$, Callewaert N: Genome sequence of the recombinant protein production host Pichia pastoris. Nat Biotechnol 2009, 27:561-566.

36. Förster J, Famili I, Fu P, Palsson BØ: Genome-scale reconstruction of the Saccharomyces cerevisiae metabolic network. Genome Res 2003, 13:244-253.

37. Mo ML, Palsson BO, Herrgard MJ: Connecting extracellular metabolomic measurements to intracellular flux states in yeast. BMC Syst Bio/ 2009, 3:37.

38. Sola A, Jouhten P, Maaheimo H, Sanchez-Ferrando F, Szyperski T, Ferrer P: Metabolic flux profiling of Pichia pastoris grown on glycerol/methanol mixtures in chemostat cultures at low and high dilution rates. Microbiology 2007, 153:281-290.

39. Verduyn C, Stouthamer AH, Scheffers WA, van Dijken JP: A theoretical evaluation of growth yields of yeasts. Antonie Van Leeuwenhoek 1991, 59:49-63.

40. Stuckrath I, Lange HC, Kotter P, van Gulik WM, Entian KD, Heijnen JJ: Characterization of null mutants of the glyoxylate cycle and gluconeogenic enzymes in S. cerevisiae through metabolic network modeling verified by chemostat cultivation. Biotechnol Bioeng 2002, 77:61-72.

41. Wriessnegger T, Gubitz G, Leitner E, Ingolic E, Cregg J, de la Cruz BJ, Daum $\mathrm{G}$ : Lipid composition of peroxisomes from the yeast Pichia pastoris grown on different carbon sources. Biochim Biophys Acta 2007, 1771:455-461.
42. Inan M, Meagher MM: Non-repressing carbon sources for alcohol oxidase (AOX1) promoter of Pichia pastoris. J Biosci Bioeng 2001, 92:585-589.

43. Trinh LB, Phue JN, Shiloach J: Effect of methanol feeding strategies on production and yield of recombinant mouse endostatin from Pichia pastoris. Biotechnol Bioeng 2003, 82:438-444.

44. Sola A, Maaheimo H, Ylonen K, Ferrer P, Szyperski T: Amino acid biosynthesis and metabolic flux profiling of Pichia pastoris. Eur $\mathrm{J}$ Biochem 2004, 271:2462-2470.

45. Kroutil W, Mang H, Edegger K, Faber K: Recent advances in the biocatalytic reduction of ketones and oxidation of sec-alcohols. Curr Opin Chem Biol 2004, 8:120-126.

46. Moore JC, Pollard DJ, Kosjek B, Devine PN: Advances in the enzymatic reduction of ketones. Acc Chem Res 2007, 40:1412-1419.

47. Goldberg K, Schroer K, Lutz S, Liese A: Biocatalytic ketone reduction--a powerful tool for the production of chiral alcohols--part I: processes with isolated enzymes. App/ Microbiol Biotechnol 2007, 76:237-248.

48. Goldberg K, Schroer K, Lutz S, Liese A: Biocatalytic ketone reduction--a powerful tool for the production of chiral alcohols-part II: whole-cell reductions. App/ Microbiol Biotechnol 2007, 76:249-255.

49. Pscheidt B, Glieder A: Yeast cell factories for fine chemical and API production. Microb Cell Fact 2008, 7:25.

50. Schroer K, Peter Luef K, Stefan Hartner F, Glieder A, Pscheidt B: Engineering the Pichia pastoris methanol oxidation pathway for improved NADH regeneration during whole-cell biotransformation. Metab Eng 2010, 12:8-17.

51. Burgard AP, Nikolaev EV, Schilling CH, Maranas CD: Flux coupling analysis of genome-scale metabolic network reconstructions. Genome Res 2004, 14:301-312.

52. Jungo C, Schenk J, Pasquier M, Marison IW, von Stockar U: A quantitative analysis of the benefits of mixed feeds of sorbitol and methanol for the production of recombinant avidin with Pichia pastoris. J Biotechnol 2007, 131:57-66.

53. Celik E, Calik P, Oliver SG: Fed-batch methanol feeding strategy for recombinant protein production by Pichia pastoris in the presence of co-substrate sorbitol. Yeast 2009, 26:473-484

54. Celik E, Calik P, Oliver SG: Metabolic flux analysis for recombinant protein production by Pichia pastoris using dual carbon sources: Effects of methanol feeding rate. Biotechnol Bioeng 2010, 105:317-329.

55. Wang Z, Wang Y, Zhang D, Li J, Hua Z, Du G, Chen J: Enhancement of cell viability and alkaline polygalacturonate lyase production by sorbitol co-feeding with methanol in Pichia pastoris fermentation. Bioresour Technol 2010, 101:1318-1323.

56. Cereghino JL, Cregg JM: Heterologous protein expression in the methylotrophic yeast Pichia pastoris. FEMS Microbiol Rev 2000, 24:45-66.

57. Takeuchi M, Kobata A: Structures and functional roles of the sugar chains of human erythropoietins. Glycobiology 1991, 1:337-346.

58. Helenius A, Aebi M: Intracellular functions of N-linked glycans. Science 2001, 291:2364-2369

59. Pratt MR, Bertozzi CR: Synthetic glycopeptides and glycoproteins as tools for biology. Chem Soc Rev 2005, 34:58-68.

60. Shelikoff M, Sinskey AJ, Stephanopoulos G: A modeling framework for the study of protein glycosylation. Biotechnol Bioeng 1996, 50:73-90.

61. Krambeck FJ, Betenbaugh MJ: A mathematical model of $\mathrm{N}$-linked glycosylation. Biotechnol Bioeng 2005, 92:711-728.

62. Liu G, Marathe DD, Matta KL, Neelamegham S: Systems-level modeling of cellular glycosylation reaction networks: O-linked glycan formation on natural selectin ligands. Bioinformatics 2008, 24:2740-2747.

63. Kim PJ, Lee DY, Jeong $\mathrm{H}$ : Centralized modularity of N-linked glycosylation pathways in mammalian cells. PLoS One 2009, 4:e7317.

64. Bielik AM, Zaia J: Historical overview of glycoanalysis. Methods Mol Biol 2010, 600:9-30

65. Taylor ME, Drickamer K: Introduction to glycobiology 2nd edition. Oxford; New York: Oxford University Press; 2006.

66. Sohn SB, Graf AB, Kim TY, Gasser B, Maurer M, Ferrer P, Mattanovich D, Lee $S Y$ : Genome-scale metabolic model of methylotrophic yeast Pichia pastoris and its use for in silico analysis of heterologous protein production. Biotechnol J 2010.

67. Hou BK, Kim JS, Jun JH, Lee DY, Kim YW, Chae S, Roh M, In YH, Lee SY: BioSilico: an integrated metabolic database system. Bioinformatics 2004, 20:3270-3272 
68. Chang A, Scheer M, Grote A, Schomburg I, Schomburg D: BRENDA, AMENDA and FRENDA the enzyme information system: new content and tools in 2009. Nucleic Acids Res 2009, 37:D588-592.

69. Bairoch A: The ENZYME database in 2000. Nucleic Acids Res 2000, 28:304-305.

70. Caspi R, Altman T, Dale JM, Dreher K, Fulcher CA, Gilham F, Kaipa P, Karthikeyan AS, Kothari A, Krummenacker M, et al.: The MetaCyc database of metabolic pathways and enzymes and the BioCyc collection of pathway/genome databases. Nucleic Acids Res 2010, 38:D473-479.

71. Kanehisa M, Goto S, Hattori M, Aoki-Kinoshita KF, Itoh M, Kawashima S, Katayama T, Araki M, Hirakawa M: From genomics to chemical genomics: new developments in KEGG. Nucleic Acids Res 2006, 34:D354-357.

72. Varma A, Boesch BW, Palsson BO: Stoichiometric interpretation of Escherichia coli glucose catabolism under various oxygenation rates. Appl Environ Microbiol 1993, 59:2465-2473.

73. Varma A, Palsson BO: Stoichiometric flux balance models quantitatively predict growth and metabolic by-product secretion in wild-type Escherichia coli W3110. Appl Environ Microbiol 1994, 60:3724-3731.

74. Raman K, Chandra N: Flux balance analysis of biological systems: applications and challenges. Brief Bioinform 2009, 10:435-449.

75. Brooke A, Kendrick D, Meeraus A, Raman R: GAMS - A user's guide. In Book GAMS - A user's quide City: GAMS Development Corporation; 1998. Editor ed.^eds.

76. Kim PJ, Lee DY, Kim TY, Lee KH, Jeong H, Lee SY, Park S: Metabolite essentiality elucidates robustness of Escherichia coli metabolism. Proc Natl Acad Sci USA 2007, 104:13638-13642.

77. Chung BK, Lee DY: Flux-sum analysis: a metabolite-centric approach for understanding the metabolic network. BMC Syst Biol 2009, 3:117.

78. Golovlev EL, Golovleva LA: Physiology of microbial cell and metabolic engineering. Microbiology 2000, 69:149-162.

79. Gurakan T, Marison IW, Von Stockar U, Gustafsson L, Gnaiger E: Proposals for a standardized sample handling procedure for the determination of elemental composition and enthalpy of combustion of biological material. Thermochim Act 1990, 172:251-266.

doi: $10.1186 / 1475-2859-9-50$

Cite this article as: Chung et al., Genome-scale metabolic reconstruction and in silico analysis of methylotrophic yeast Pichia pastoris for strain improvement Microbial Cell Factories 2010, 9:50

Submit your next manuscript to BioMed Centra and take full advantage of:

- Convenient online submission

- Thorough peer review

- No space constraints or color figure charges

- Immediate publication on acceptance

- Inclusion in PubMed, CAS, Scopus and Google Scholar

- Research which is freely available for redistribution

Submit your manuscript at www.biomedcentral.com/submit
C Biomed Central 